\title{
$\therefore$ An Unusual Long Standing Foreign Body in the Left Bronchus of 6 Year Old Boy - A Case Report
}

\section{IJCRR}

Section: Healthcare ISI Impact Factor (2019-20): 1.628

IC Value (2019): 90.81 $\operatorname{SJIF}(2020)=7.893$

(c) (7) (8)

Copyright@IJCRR

\section{Swain SK ${ }^{1}$, Swain $\mathbf{S}^{2}$, Acharya $\mathbf{S}^{1}$}

\author{
'Professor, Department of Otorhinolaryngology, IMS and SUM hospital, Siksha "O” Anusandhan University, K8, Kalinganagar, \\ Bhubaneswar-751003, Odisha, India; 'Assistant Professor, Department of Anesthesiology, IMS and SUM hospital, Siksha "O” Anusandhan \\ University, K8, Kalinganagar, Bhubaneswar-751003, Odisha, India.
}

\section{ABSTRACT}

Introduction: Inhalation of foreign body (FB) into the airway is considered a serious problem in a pediatric patient. Children of less attention by parents at an early age are more vulnerable to inhalation of FB. A long-standing undiagnosed FB such as whistle in bronchus is very rare and lethal.

Case Report: A 6-year-old boy was presenting with a mild dry cough for 2 months. He had no evidence of breathing difficulty. He developed occasional whistling sounds during sleep for 5 days.

Result: The FB was confirmed by computed tomography (CT) scan of the thorax and was removed successfully by rigid bronchoscopy under general anaesthesia without any complications.

Conclusion: The diagnosis of FB in the bronchus is often difficult because of lack of parental ignorance and the absence of clear history. FB inhalation is often associated with significant morbidity.

Key Words: Foreign body, Inhalation, Bronchus, Long-standing, Whistle, Rigid Bronchoscopy

\section{INTRODUCTION}

A foreign body (FB) is a substance or an object foreign to the site where it is found. ${ }^{1}$ When FB enters the airway and results in choking, called FB aspiration. The FBs can enter the airway via mouth or nose and lodge any location from supraglottis to the terminal bronchioles. ${ }^{2} \mathrm{FB}$ aspirations is a commonly encountered potential life-threatening situation in the pediatric age group. ${ }^{2}$ Near-total obstructions of the laryngotracheal airway by FB can result in immediate asphyxia and death. FB in the airway is always a challenge to the clinicians for diagnosis and treatment. There are different types of FBs in the airway such as organic, inorganic, metallic, and nonmetallic. The majority of the FBs in the airway are organic, mainly food materials. The common organic FBs of the airway are nuts, seeds, foods, and bone pieces. ${ }^{3}$ The inorganic FBs are plastic beads, caps of pens, coins, pills, nails, teeth, pins and plastic materials. ${ }^{3}$ The plastic whistle is an inert FB found in the bronchus. Prevention and prompt diagnosis can be life-saving. The complications of airway FBs depend on the shape, size, nature and duration of the FB. ${ }^{4}$ Although FB inhalation in the airway has been de- scribed for many years, undiagnosed and unsuspected FBs are still found in the airway leading to several complications and make threatening to the life because of the delayed diagnosis. Here, we are presenting a case of an overlooked and long-standing FB (whistle) in the left bronchus in a 6-yearold boy.

\section{CASE REPORT}

A 6-year-old boy attended the outpatient department with complaints of mild dry and irritating cough for two months. $\mathrm{He}$ had no evidence of breathing difficulty. He was also presented with a typical symptom of whistling sound during sleep for the last 5 days. He had received several antibiotics from local paediatricians but was not relieved from the dry and irritating cough. On routine history taking at the outpatient department of Otorhinolaryngology, the child and his parents were unable to inform about the ingestion or inhalation of any FB. Clinical examination of the patient showed an average body built with a pulse rate of $88 / \mathrm{min}$ $\mathrm{BP}$ of $110 / 70 \mathrm{mmHg}$, respiratory rate $20 / \mathrm{min}$. Examination

\section{Corresponding Author:}

Prof. Santosh Kumar Swain, Department of Otorhinolaryngology, IMS\&SUM Hospital, Siksha “O” Anusandhan University, K8, Kalinganagar, Bhubaneswar-751003, Odisha, India; Cell: +91-9556524887; Email: santoshswain@soa.ac.in

ISSN: 2231-2196 (Print)

Received: 12.01 .2021
ISSN: 0975-5241 (Online)

Revised: 14.02 .2021
Accepted: 10.03 .2021
Published: 26.05 .2021 
of the chest revealed a wheeze and coarse crackles in the left lower anterior chest. The rest of the general physical and systemic examinations was unremarkable. CT scan of the thorax revealed normal both sides of the lungs except a rim of the opacity in the left bronchus approximately $2.5 \mathrm{~cm}$ below the carina (Fig.1). The lumen of the left bronchus was patent and no evidence of the collapse of the lungs on the left side. Although there was no history of FB inhalation, minimal clinical symptoms and radiological tests (CT scan) gave the strong possibility for FB in the left bronchus. The plastic whistle (Fig.2) was removed by the rigid bronchoscopy along with optical forceps under general anaesthesia. There were no complications during the rigid bronchoscopy under general anaesthesia with spontaneous ventilation where propofol is combined with midazolam, fentanyl, dexamethasone, and sevoflurane. The child was discharged with no sequelae on day 3 of hospitalization.

\section{DISCUSSION}

Inhalation of the FB in the airway is a potentially life-threatening situation. There was high morbidity and mortality by airway FB before the introduction of bronchoscopy. After the advent of the bronchoscope, this morbidity and mortality were reduced drastically. The first case of FB removal by bronchoscopy from a bronchus was done by Gustav Killian, a German Otolaryngologist in $1897 .{ }^{5} \mathrm{FB}$ in the bronchus is more common in the pediatric age than the adult population. The peak incidence of FB aspiration in the pediatric age is during the second year of life and the sixth decade of the adult population. ${ }^{6} \mathrm{FB}$ inhalation commonly found in boys, with an overall sex ratio from 2.1:1 to 2.6:1. ${ }^{7}$ Majority of the FB inhalation, affects the boys which could be explained by their likely more impulsive nature and interest in adventurous games. However, contrary to this trend, there is a similar incidence of FB inhalation in both genders. ${ }^{8}$ The most common location for FB aspiration in the adult age group is the right bronchus as it is more vertical disposition whereas it is a central location in the majority of cases of children. ${ }^{9}$ The common vegetable FBs include nuts and grains which cause acute inflammation than metallic, plastic, or bony FBs. In this case, the FB was a plastic whistle with a lumen for the flow of air. The most common symptoms in the case of FB aspiration of the lungs are called penetration syndrome which means the sudden onset of choking and intractable cough with or without vomiting. The typical severe clinical presentation of patients with airway FB is dyspnea. In this case, the child had no complaints of any clinical symptoms at the time of the inhalation of the whistle except occasional mild dry cough. Our case also presented with a typical symptom of whistling sound during sleep for the last 5 days. If the FB stays for a longer period in situ, the higher the chance of granulation tissue formation, leading to a smaller lumen and symptoms usually become more pronounced. In the case of long-standing neglected FB in the bronchus, there may be inflammation, pulmonary inflammation, lung collapse, abscess formation in the lungs and even malignant transformation. ${ }^{10}$ The longstanding FB in the tracheobronchial airway may result in pneumonia, bronchiectasis, lung abscess, atelectasis, post-obstructive hyperinflation, occasionally misdiagnosed as bronchial asthma or lung malignancy in adult age who has inhaled an FB. ${ }^{11}$ The response of the tissue of laryngotracheobronchitis airway to the $\mathrm{FB}$ varies as per the composition of the FB and associated infections. The relative inert nature of the plastic material such as the whistle as in our case produces a mild inflammatory reaction and promotes to keep in the laryngotracheal airway for a longer period. The diagnosis of the FB such as plastic whistle in airway often delayed because its diagnosis ranges from hours to years and is the considerably lesser duration in pediatric patients than adult age group. ${ }^{6}$

Our patient had 2 months delay in diagnosis because of the asymptomatic patient and the incidence of FB inhalation was not known to the parents. FB aspiration often mimics certain diseases such as bronchial asthma, anaphylaxis, angioedema, acute exacerbation of COPD, central airway obstruction, congestive heart failure, drug reaction, and vocal fold dysfunction. ${ }^{12}$ Central airway obstructions are usually caused by malignancy of the lungs. The metastatic lesions from the thyroid, breast, colon, kidney, and melanoma can obstruct the central airways. ${ }^{13}$ The clinical presentations of delayed FBs may mimic bronchial asthma, pneumonia, croup, and even gastroesophageal reflux disease.

Chest X-ray is often helpful to find out the site of FB but difficult to provide a conclusion in the case of non-metallic FB. In chest X-ray, the non-radio-opaque FB may be aided by inspiration-expiration films, which show air trapping distal to the blocked segment of the bronchus. ${ }^{14}$ However, a chest X-ray does not detect approximately $80 \%$ of the FBs. ${ }^{15}$ So, a CT scan of the thorax is helpful to locate the exact site of FB. $\mathrm{CT}$ virtual bronchoscopy is a noninvasive procedure that can be helpful for an internal view of the trachea and major bronchi by three-dimensional reconstruction.

Bronchoscopy is helpful to directly detect and remove the FBs. Proper history taking, preoperative imaging, and early intervention by a skilled bronchoscopist with help of skilled anesthetists are often helpful for managing the FB bronchus or airway. The successful outcome of FB removal from the airway depends on several factors such as the age of the patient, clinical presentations, and the skill of the surgeons and anaesthetists. Early diagnosis, prompt treatment, vigilant monitoring, and very good cooperation between surgeon and anaesthetist are of paramount importance for performing rigid bronchoscopy in a pediatric patient. Spontaneous ventilation is often the preferred technique where propofol 
is combined with midazolam, fentanyl, dexamethasone, and sevoflurane for effective anaesthesia in the pediatric patient during rigid bronchoscopy. ${ }^{16}$ The confirmation for the presence of FB in the airway and its removal is done by bronchoscopy. Rigid bronchoscopy is often used for the removal of the FB from the airway. However, a flexible bronchoscope may be used for the removal of the tracheobronchial FBs. Better instrumentations and visualization is helpful to decrease the complication rates during bronchoscopy. The use of optical forceps is highly helpful for the removal of airway FBs. Virtual bronchoscopy can aid in the diagnosis of the laryngotracheal FB. The airway FB can be prevented by providing proper education to the public and parents. They should be taught about the dangers of this accidental inhalation and its eventualities.

\section{CONCLUSION}

Inhalation of FB is a preventable mishap. FB inhalation in the airway is a potentially lethal event. A long-standing $\mathrm{FB}$ in the bronchus is an uncommon clinical condition and overlooked for a longer period in this case. The importance of the early diagnosis and rarity of long-standing FB in the left bronchus is stressed here. Asymptomatic airway FB is always considered challenging for clinicians. FB aspiration can be prevented by imparting proper awareness or education to the parents and public at large. They should be informed about the dangers of these accidents and the eventualities of this lethal problem. Optimum care should be given to the children for preventing FB aspiration by parents. Timely intervention with an experienced surgical team can reduce complications and mortality. Currently, CT virtual bronchoscopy is a non-invasive technique that can provide an internal view of the trachea and major bronchi by three-dimensional reconstruction and is helpful for easy removal of foreign body bronchus.

\section{Acknowledgement: None}

Conflict of interest: Nil

Funding: Nil

Author Contribution: Prof.Santosh Kumar Swain: Concept, data collection and data analysis; Dr.Sumita Swain:
Data collection and drafting; Dr.Satyabrata Acharya: Data collection

\section{REFERENCES}

1. Swain SK, Mohanty S, Behera IC.An unsafe foreign body at an unsafe site of the airway.Int J Phonosurg Laryngol 2014;4(1):1719.

2. Kumar S, Al-Abri R, Sharma A, Al-Kindi H, Mishra P. Management of pediatric tracheobronchial foreign body aspiration. Oman Med J2010;25(4): 25;17-19.

3. Saki N, Nikakhlagh S, Rahim F, Abshirini H. Foreign body aspirations in infancy: a 20-year experience. Int $\mathrm{J}$ Med sci 2009;6(6):322-328.

4. Swain SK, Panigrahi R, Mishra S, C.Sundaray. An unusual longstanding tracheal foreign body - A rare incidence.Egypt J of Ear, Nose, Throat and Alli Sc 2015;16(1):91-93.

5. Clerf LH. Historical aspects of foreign bodies in the air and food passages. Ann Otol Rhinol Laryngol 1952;61(1):5-17.

6. Swain SK, Mallik KC. Ear, nose, and throat foreign bodies in pediatric age. Med J Babylon 2020;17(3):238-243.

7. Ding G, Wu B, Vinturache A, Cai C, Lu M, Gu H. Tracheobronchial foreign body aspiration in children: A retrospective singlecentre cross-sectional study. Medicine2020;99(22):e20480.

8. Friedman EM.Tracheobronchial foreign bodies. Otolaryngol Clin North Am 2000;33(1):179-185.

9. $\mathrm{Mu} \mathrm{L}, \mathrm{He}$ P, Sun D.Inhalation of foreign bodies in Chinese children: Review of 400 cases.Laryngoscope 1991;101(6):657-660.

10. Miladinovi CA, Hajdarevi CB. End bronchial foreign bodies cause bronchial carcinoma. Plucne Bolesti 1971;23(1):344-346.

11. Swain SK, Sahoo S, Sahu MC. From tooth extraction to fatal airway complication in a child-A case Report. Egypt J Ear Nose Throat Allied sci2016;17(1):27-29.

12. Kann K, Long B, Koyfman A. Clinical mimics an emergency medicine-focused review of asthma mimics. J Emerg Med2017; 53 (2): 195-201.

13. Murgu SD, Egressy K, Laxmanan B, Doblare G, Ortiz-Comino R, Hogarth DK. Central airway obstruction: benign strictures, tracheobronchomalacia, and malignancy-related obstruction. Chest. 2016;150(2):426-441.

14. Cherrez-Ojeda I, Felix M, Vanegas E, Mata VL, Jimenez FM, Fornell LG. Rhonchus and Valve-Like Sensation as Initial Manifestations of Long-Standing Foreign Body Aspiration: A Case Report. Am J Case Rep. 2019;20(1):70-73.

15. Swain SK, Mohanty S, Sahu MC, Behera IC. Safe and effective anaesthesia during paediatric rigid bronchoscopy: An experience at a tertiary care centre of Eastern India. Pediatr Pol. 2015;90(6):470-473. 


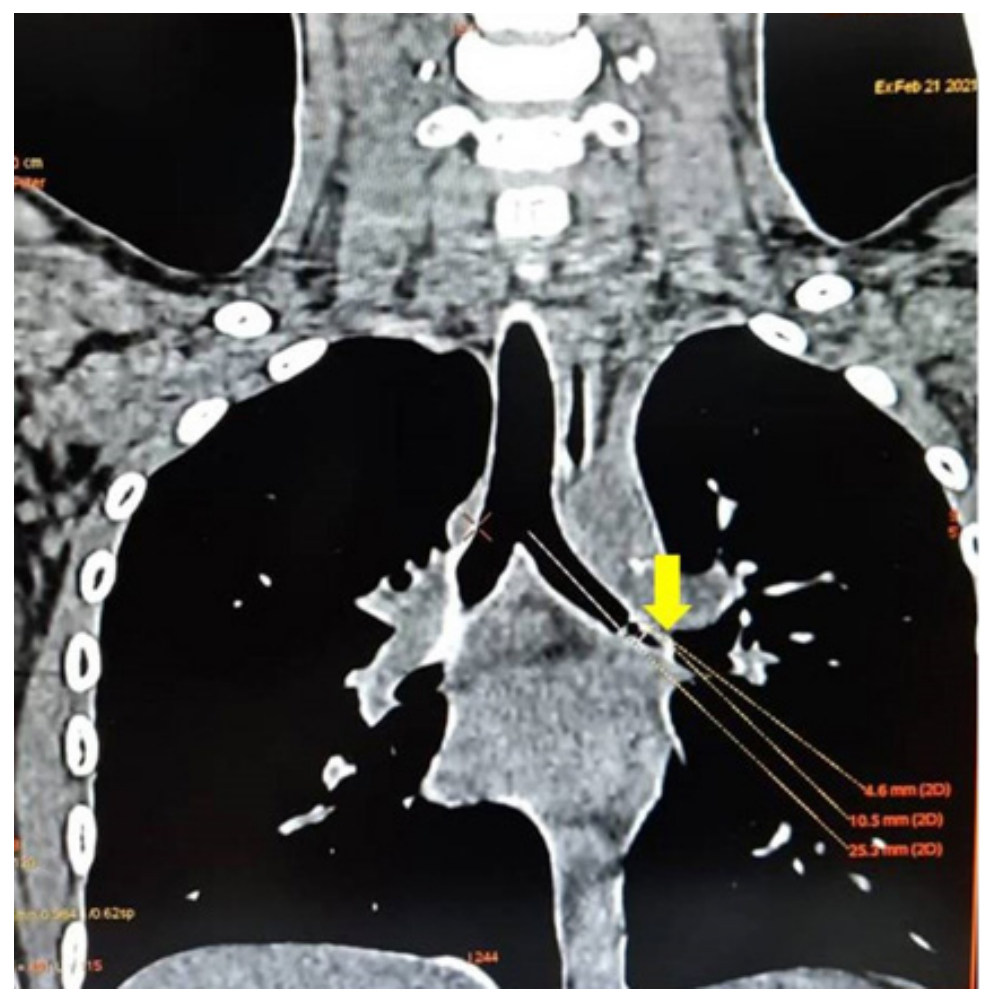

Figure 1: CT scan of the thorax showing a tubular structure with rim of opacity in the left bronchus (Yellow arrow).

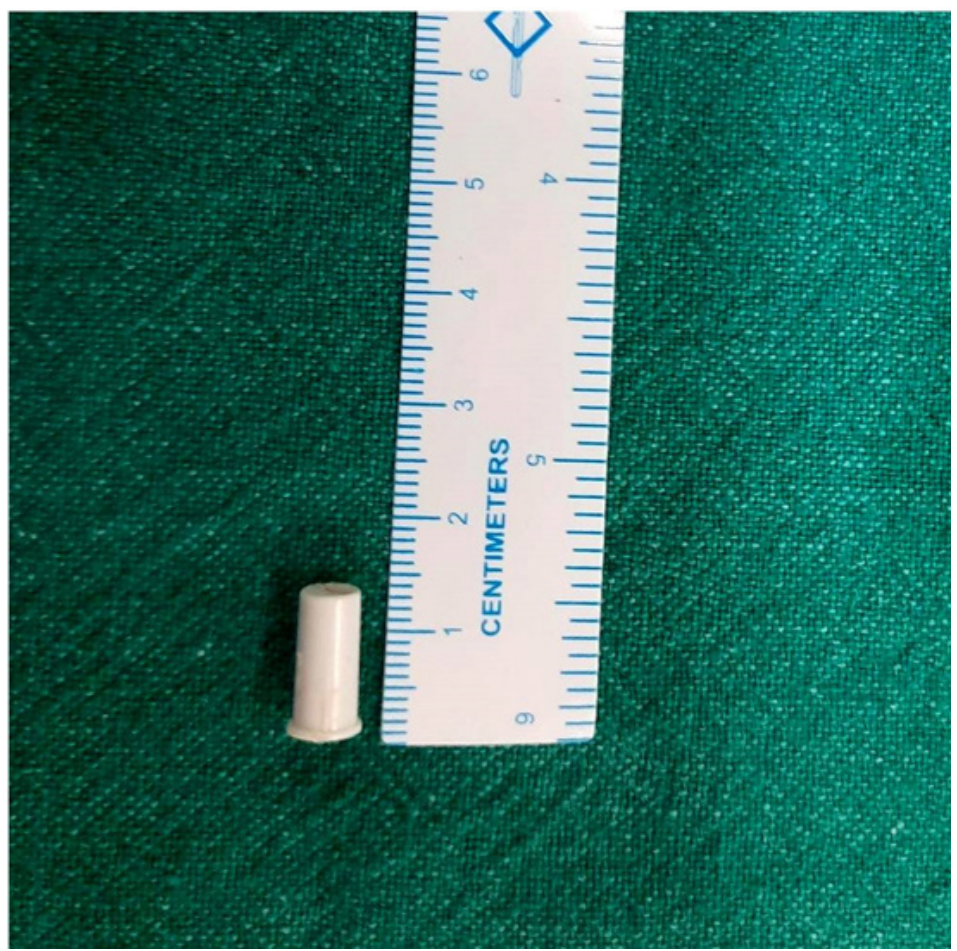

Figure 2: A whistle removed from the left bronchus. 